\title{
Almost weak continuity for multifunctions in ideal topological spaces
}

\author{
CHAWALIT BOONPOK, CHOKCHAI VIRIYAPONG \\ Mathematics and Applied Mathematics Research Unit \\ Department of Mathematics, Faculty of Science, Mahasarakham University \\ Maha Sarakham, 44150 \\ THAILAND
}

\begin{abstract}
The main goal of this paper is to introduce the concepts of upper and lower almost weakly $\star$-continuous multifunctions. Some characterizations of upper and lower almost weakly $\star$-continuous multifunctions are investigated.
\end{abstract}

Key-Words: $\star$-open set, upper almost weakly $\star$-continuous multifunction, lower almost weakly $\star$-continuous multifunction

Received: January 21, 2020. Revised: May 15, 2020. Accepted: July 6, 2020. Published: July 20, 2020.

\section{Introduction}

Continuity is an important concept for the study and investigation in topological spaces. The concept of weak continuity due to Levine [16] is one of the most important weak forms of continuity in topological spaces. Ekici et al. [8] established a new class of functions called weakly $\lambda$-continuous functions which is weaker than $\lambda$-continuous functions and investigated some fundamental properties of weakly $\lambda$ continuous functions. Popa and Noiri [19] introduced a new notion of weakly $(\tau, m)$-continuous functions as functions from a topological space into a set satisfying some minimal conditions and obtained some characterizations and several properties of such functions. Janković [14] defined almost weakly continuous functions as a generalization of both weakly continuous functions and almost continuous functions in the sense of Husain [12]. Smithson [22] and Popa [20, 21] extended independently these concepts to multifunctions and introduced upper (resp. lower) weakly continuous multifunctions and upper (resp. lower) almost continuous multifunctions. The present authors introduced and studied other weak forms of continuous multifunctions: weakly quasicontinuous multifunctions, almost weakly continuous multifunctions, weakly $\alpha$-continuous multifunctions and weakly $\beta$-continuous multifunctions. These multifunctions have similar properties. Noiri and Popa [18] have defined and investigated the notion of almost weakly continuous multifunctions. Ekici at al. [7] introduced the concept of almost contra-continuous multifunctions and investigated several characterizations of almost contra-continuous multifunctions. In [17], the present authors introduced the concept of upper (resp. lower) almost $m$-continuous multifunctions as multifunctions from a set satisfying some minimal conditions into a topological space and obtained some characterizations of such multifunctions.

The concept of ideals in topological spaces has been introduced and studied by Kuratowski [15] and Vaidyanathswamy [23]. Every topological space is an ideal topological space and all the results of ideal topological spaces are generalizations of the results established in topological spaces. In 1990, Janković and Hamlett [13] introduced the notion of $\mathscr{I}$-open sets in ideal topologial spaces. Abd El-Monsef et al. [1] further investigated $\mathscr{I}$-open sets and $\mathscr{I}$ continuous functions. Later, several authors studied ideal topological spaces giving several convenient definitions. Some authors obtained decompositions of continuity. For instance, Açikgöz et al. [2] introduced and investigated the notions of weakly$\mathscr{I}$-continuous and weak ${ }^{\star}-\mathscr{I}$-continuous functions in ideal topological spaces. Donthev [6] introduced the notion of per- $\mathscr{I}$-open sets and obtained a decomposition of $\mathscr{I}$-continuity. In [11], the present authors introduced the notions of semi- $\mathscr{I}$-open sets, $\alpha-\mathscr{I}$ open sets and $\beta-\mathscr{I}$-open sets via idealization and using these sets obtained new decompositions of continuity. Hatir and Noiri [10] investigated further properties of semi- $\mathscr{I}$-open sets and semi- $\mathscr{I}$-continuity. Moreover, the present authors [9] introduced and investigated the notions of strong $\beta-\mathscr{I}$-open sets and strongly $\beta-\mathscr{I}$-continuous functions.

The purpose of the present paper is to introduce the notions of upper and lower almost weakly $\star-$ continuous multifunctions as a multifunction from an ideal topological space into an ideal topological space and investigate several characterizations of such multifunctions. 


\section{Preliminaries}

Throughout the present paper, spaces $(X, \tau)$ and $(Y, \sigma)$ (or simply $X$ and $Y$ ) always mean topological spaces on which no separation axioms are assumed unless explicitly stated. In a topological space $(X, \tau)$, the closure and the interior of any subset $A$ of $X$ will denoted by $\mathrm{Cl}(A)$ and $\operatorname{Int}(A)$, respectively. An ideal $\mathscr{I}$ on a topological space $(X, \tau)$ is a nonempty collection of subsets of $X$ satisfying the following properties: (1) $A \in \mathscr{I}$ and $B \subseteq A$ implies $B \in \mathscr{I}$; (2) $A \in \mathscr{I}$ and $B \in \mathscr{I}$ implies $A \cup B \in \mathscr{I}$. A topological space $(X, \tau)$ with an ideal $\mathscr{I}$ on $X$ is called an ideal topological space and is denoted by $(X, \tau, \mathscr{I})$. For an ideal topological space $(X, \tau, \mathscr{I})$ and a subset $A$ of $X, A^{\star}(\mathscr{I})$ is defined as follows: $A^{\star}(\mathscr{I})=\{x \in X: U \cap A \notin \mathscr{I}$ for every open neighbourhood $U$ of $x\}$. In case there is no chance for confusion, $A^{\star}(\mathscr{I})$ is simply written as $A^{\star}$. In [15], $A^{\star}$ is called the local function of $A$ with respect to $\mathscr{I}$ and $\tau$ and $\mathrm{Cl}^{\star}(A)=A^{\star} \cup A$ defines a Kuratowski closure operator for a topology $\tau^{\star}(\mathscr{I})$. For every ideal topological space $(X, \tau, \mathscr{I})$, there exists a topology $\tau^{\star}(\mathscr{I})$ finer than $\tau$, generated by $\mathscr{B}(\tau, \mathscr{I})=\left\{U-I_{0} \mid U \in \tau\right.$ and $\left.I_{0} \in \mathscr{I}\right\}$, but in general $\mathscr{B}(\tau, \mathscr{I})$ is not always a topology [13]. A subset $A$ is said to be $\star$-closed [13] if $A^{\star} \subseteq A$. The complement of a $\star$-closed set is called $\star$-open. The interior of a subset $A$ in $\left(X, \tau^{\star}(\mathscr{I})\right)$ is denoted by $\operatorname{Int}^{\star}(A)$.

By a multifunction $F: X \rightarrow Y$, we mean a pointto-set correspondence from $X$ into $Y$, and we always assume that $F(x) \neq \emptyset$ for all $x \in X$. For a multifunction $F: X \rightarrow Y$, following [3], we shall denote the upper and lower inverse of a set $B$ of $Y$ by $F^{+}(B)$ and $F^{-}(B)$, respectively, that is,

$$
F^{+}(B)=\{x \in X \mid F(x) \subseteq B\}
$$

and $F^{-}(B)=\{x \in X \mid F(x) \cap B \neq \emptyset\}$. In particular, $F^{-}(y)=\{x \in X \mid y \in F(x)\}$ for each point $y \in Y$. For each $A \subseteq X, F(A)=\cup_{x \in A} F(x)$. Then $F$ is said to be surjection if $F(X)=Y$, or equivalent, if for each $y \in Y$ there exists $x \in X$ such that $y \in F(x)$ and $F$ is called injection if $x \neq y$ implies $F(x) \cap F(y)=\emptyset$.

Definition 1. [6] A subset $A$ of an ideal topological space $(X, \tau, \mathscr{I})$ is said to be:

(1) pre- $\mathscr{I}$-open if $A \subseteq \operatorname{Int}\left(\mathrm{Cl}^{\star}(A)\right)$;

(2) pre- $\mathscr{I}$-closed if its complement is pre- $\mathscr{I}$-open.

For a subset $A$ of an ideal topological space $(X, \tau, \mathscr{I})$, the intersection of all pre- $\mathscr{I}$-closed sets of $X$ containing $A$ is called the pre- $\mathscr{I}$-closure [24] of $A$ and is denoted by $p \imath \mathrm{Cl}(A)$. The union of all pre$\mathscr{I}$-open sets of $X$ contained in $A$ is called the pre$\mathscr{I}$-interior of $A$ and is denoted by $p \imath \operatorname{Int}(A)$.
Lemma 2. [24] Let A be a subset of an ideal topological space $(X, \tau, \mathscr{I})$ and $x \in X$. Then, the following properties hold:

(1) $x \in \operatorname{p\imath Cl}(A)$ if and only if $U \cap A \neq \emptyset$ for every pre- $\mathscr{I}$-open set $U$ of $X$ containing $x$.

(2) $A$ is pre- $\mathscr{I}$-closed if and only if $A=\operatorname{plCl}(A)$.

(3) $\operatorname{p\imath Cl}(X-A)=X-\operatorname{pint}(A)$.

(4) $\operatorname{prInt}(X-A)=X-p \imath C l(A)$.

Lemma 3. For a subset $A$ of an ideal topological space $(X, \tau, \mathscr{I})$, the following properties hold:

(1) $\operatorname{p\imath Cl}(A)=A \cup C l\left(\operatorname{Int^{\star }}(A)\right)$ [25].

(2) $\operatorname{pl\operatorname {Int}}(A)=A \cap \operatorname{Int}\left(C l^{\star}(A)\right)$.

\section{Some characterizations}

In this section, we introduce the notions of upper and lower almost weakly $\star$-continuous multifunctions. Moreover, several interesting characterizations of upper and lower almost weakly $\star$-continuous multifunctions are investigated.

Definition 4. A multifunction $F:(X, \tau, \mathscr{I}) \rightarrow$ $(Y, \sigma, \mathscr{J})$ is said to be:

(1) upper almost weakly $\star$-continuous iffor each $x \in$ $X$ and each $\star$-open set $V$ of $Y$ such that $F(x) \subseteq$ $V, x \in \operatorname{Int}\left(C l^{\star}\left(F^{+}\left(C l^{\star}(V)\right)\right)\right)$;

(2) lower almost weakly $\star$-continuous iffor each $x \in$ $X$ and each $\star$-open set $V$ of $Y$ such that

$$
\begin{array}{r}
F(x) \cap V \neq \emptyset, \\
x \in \operatorname{Int}\left(C l^{\star}\left(F^{-}\left(C l^{\star}(V)\right)\right)\right) .
\end{array}
$$

The following results give some characterizations of upper and lower almost weakly $\star$-continuous multifunctions.

Theorem 5. For a multifunction $F:(X, \tau, \mathscr{I}) \rightarrow$ $(Y, \sigma, \mathscr{J})$, the following properties are equivalent:

(1) $F$ is upper almost weakly $\star$-continuous;

(2) $F^{+}(V) \subseteq \operatorname{Int}\left(C l^{\star}\left(F^{+}\left(C l^{\star}(V)\right)\right)\right)$ for every $\star-$ open set $V$ of $Y$;

(3) $C l\left(I n t^{\star}\left(F^{-}(V)\right)\right) \subseteq F^{-}\left(C l^{\star}(V)\right)$ for every $\star-$ open set $V$ of $Y$;

(4) $\operatorname{p\imath Cl}\left(F^{-}(V)\right) \subseteq F^{-}\left(C l^{\star}(V)\right)$ for every $\star$-open set $V$ of $Y$;

(5) $F^{+}(V) \subseteq p u \operatorname{Int}\left(F^{+}\left(C l^{\star}(V)\right)\right)$ for every $\star$-open set $V$ of $Y$; 
(6) for each $x \in X$ and each $\star$-open set $V$ of $Y$ containing $F(x)$, there exists a pre- $\mathscr{I}$-open set $U$ of $X$ containing $x$ such that $F(U) \subseteq C l^{\star}(V)$.

Proof. (1) $\Rightarrow(2)$ : Let $V$ be any $\star$-open set of $Y$ and $x \in F^{+}(V)$. Then $F(x) \subseteq V$. Since $F$ is upper almost weakly $\star$-continuous,

$$
x \in \operatorname{Int}\left(\mathrm{Cl}^{\star}\left(F^{+}\left(\mathrm{Cl}^{\star}(V)\right)\right)\right) .
$$

Consequently, we obtain

$$
F^{+}(V) \subseteq \operatorname{Int}\left(\mathrm{Cl}^{\star}\left(F^{+}\left(\mathrm{Cl}^{\star}(V)\right)\right)\right) .
$$

$(2) \Rightarrow(3)$ : Let $V$ be any $\star$-open set of $Y$. Since $Y-\mathrm{Cl}^{\star}(V)$ is $\star$-open and by $(2)$, we have

$$
\begin{aligned}
X-F^{-}\left(\mathrm{Cl}^{\star}(V)\right) & =F^{+}\left(Y-\mathrm{Cl}^{\star}(V)\right) \\
& \subseteq \operatorname{Int}\left(\mathrm{Cl}^{\star}\left(F^{+}\left(\mathrm{Cl}^{\star}\left(Y-\mathrm{Cl}^{\star}(V)\right)\right)\right)\right) \\
& =\operatorname{Int}\left(\mathrm{Cl}^{\star}\left(F^{+}\left(Y-\operatorname{Int}^{\star}\left(\mathrm{Cl}^{\star}(V)\right)\right)\right)\right) \\
& \subseteq \operatorname{Int}\left(\mathrm{Cl}^{\star}\left(F^{+}(Y-V)\right)\right) \\
& =\operatorname{Int}\left(\mathrm{Cl}^{\star}\left(X-F^{-}(V)\right)\right) \\
& =X-\mathrm{Cl}\left(\operatorname{Int}^{\star}\left(F^{-}(V)\right)\right)
\end{aligned}
$$

and hence $\mathrm{Cl}\left(\operatorname{Int}^{\star}\left(F^{-}(V)\right)\right) \subseteq F^{-}\left(\mathrm{Cl}^{\star}(V)\right)$.

$(3) \Rightarrow(4)$ : Let $V$ be any $\star$-open set of $Y$. By (3) and Lemma $3(1)$,

$$
\begin{aligned}
p \imath \mathrm{Cl}\left(F^{-}(V)\right) & =F^{-}(V) \cup \mathrm{Cl}\left(\operatorname{Int}^{\star}\left(F^{-}(V)\right)\right) \\
& \subseteq F^{-}\left(\mathrm{Cl}^{\star}(V)\right) \cup \mathrm{Cl}\left(\operatorname{Int}^{\star}\left(F^{-}(V)\right)\right) \\
& =F^{-}\left(\mathrm{Cl}^{\star}(V)\right) .
\end{aligned}
$$

$(4) \Rightarrow(5)$ : Let $V$ be any $\star$-open set of $Y$. Since $Y-\mathrm{Cl}^{\star}(V)$ is $\star$-open and by $(4)$, we have

$$
\begin{aligned}
X-p \imath \operatorname{Int}\left(F^{+}\left(\mathrm{Cl}^{\star}(V)\right)\right) & =p \imath \mathrm{Cl}\left(X-F^{+}\left(\mathrm{Cl}^{\star}(V)\right)\right) \\
& =p \imath \mathrm{Cl}\left(F^{-}\left(Y-\mathrm{Cl}^{\star}(V)\right)\right) \\
& \subseteq F^{-}\left(\mathrm{Cl}^{\star}\left(Y-\mathrm{Cl}^{\star}(V)\right)\right) \\
& \subseteq F^{-}\left(\mathrm{Cl}^{\star}(Y-V)\right) \\
& =F^{-}(Y-V) \\
& =X-F^{+}(V)
\end{aligned}
$$

and hence $F^{+}(V) \subseteq p \imath \operatorname{Int}\left(F^{+}\left(\mathrm{Cl}^{\star}(V)\right)\right)$.

$(5) \Rightarrow(6)$ : Let $x \in X$ and $V$ be any $\star$-open set of $Y$ containing $F(x)$. By (5), we have

$$
x \in F^{+}(V) \subseteq p \imath \operatorname{Int}\left(F^{+}\left(\mathrm{Cl}^{\star}(V)\right)\right)
$$

and so there exists a pre- $\star$-open set $U$ of $X$ containing $x$ such that $U \subseteq F^{+}\left(\mathrm{Cl}^{\star}(V)\right)$; hence

$$
F(U) \subseteq \mathrm{Cl}^{\star}(V) .
$$

$(6) \Rightarrow(1)$ : Let $x \in X$ and $V$ be any $\star$-open set of $Y$ containing $F(x)$. By (6), there exists a pre- $\mathscr{I}$ open set $U$ of $X$ containing $x$ such that

$$
F(U) \subseteq \mathrm{Cl}^{\star}(V) .
$$

Thus, $U \subseteq F^{+}\left(\mathrm{Cl}^{\star}(V)\right)$ and hence

$$
x \in U \subseteq \operatorname{Int}\left(\mathrm{Cl}^{\star}(U)\right) \subseteq \operatorname{Int}\left(\mathrm{Cl}^{\star}\left(F^{+}\left(\mathrm{Cl}^{\star}(V)\right)\right)\right) .
$$

This shows that $F$ is upper almost weakly $\star-$ continuous.

Theorem 6. For a multifunction $F:(X, \tau, \mathscr{I}) \rightarrow$ $(Y, \sigma, \mathscr{J})$, the following properties are equivalent:

(1) F is lower almost weakly $\star-c o n t i n u o u s ;$

(2) $F^{-}(V) \subseteq \operatorname{Int}\left(C l^{\star}\left(F^{-}\left(C l^{\star}(V)\right)\right)\right)$ for every $\star-$ open set $V$ of $Y$;

(3) $C l\left(\operatorname{Int}^{\star}\left(F^{+}(V)\right)\right) \subseteq F^{+}\left(C l^{\star}(V)\right)$ for every $\star$ open set $V$ of $Y$;

(4) $\operatorname{p\imath Cl}\left(F^{+}(V)\right) \subseteq F^{+}\left(C l^{\star}(V)\right)$ for every $\star$-open set $V$ of $Y$;

(5) $F^{-}(V) \subseteq \operatorname{puInt}\left(F^{-}\left(C l^{\star}(V)\right)\right)$ for every $\star$-open set $V$ of $Y$;

(6) for each $x \in X$ and each $\star$-open set $V$ of $Y$ such that $F(x) \cap V \neq \emptyset$, there exists a pre- $\mathscr{I}$-open set $U$ of $X$ containing $x$ such that

$$
F(z) \cap C l^{\star}(V) \neq \emptyset
$$

for each $z \in U$.

Proof. The proof is similar to that of Theorem 5 .

Theorem 7. For a multifunction $F:(X, \tau, \mathscr{I}) \rightarrow$ $(Y, \sigma, \mathscr{J})$, the following properties are equivalent:

(1) F is upper almost weakly $\star$-continuous;

(2) $C l\left(\operatorname{Int}^{\star}\left(F^{-}\left(\operatorname{Int} t^{\star}(K)\right)\right)\right) \subseteq F^{-}(K)$ for every $\star-$ closed set $K$ of $Y$;

(3) $\operatorname{prCl}\left(F^{-}\left(\operatorname{Int} t^{\star}(K)\right)\right) \subseteq F^{-}(K)$ for every $\star-$ closed set $K$ of $Y$;

(4) $\mathrm{plCl}\left(F^{-}\left(\operatorname{Int}^{\star}\left(C l^{\star}(B)\right)\right)\right) \subseteq F^{-}\left(C l^{\star}(B)\right)$ for every subset $B$ of $Y$;

(5) $F^{+}\left(\operatorname{Int} t^{\star}(B)\right) \subseteq p \operatorname{pInt}\left(F^{+}\left(C l^{\star}\left(\operatorname{Int} t^{\star}(B)\right)\right)\right)$ for every subset $B$ of $Y$. 
Proof. (1) $\Rightarrow(2)$ : Let $K$ be any $\star$-closed set of $Y$. Then $Y-K$ is $\star$-open in $Y$ and by Theorem 5 , we have

$$
\begin{aligned}
X-F^{-}(K) & =F^{+}(Y-K) \\
& \subseteq \operatorname{Int}\left(\mathrm{Cl}^{\star}\left(F^{+}\left(\mathrm{Cl}^{\star}(Y-K)\right)\right)\right) \\
& =\operatorname{Int}\left(\mathrm{Cl}^{\star}\left(F^{+}\left(Y-\operatorname{Int}^{\star}(K)\right)\right)\right) \\
& =\operatorname{Int}\left(\mathrm{Cl}^{\star}\left(X-F^{-}\left(\operatorname{Int}^{\star}(K)\right)\right)\right) \\
& =X-\operatorname{Cl}\left(\operatorname{Int}^{\star}\left(F^{-}\left(\operatorname{Int}^{\star}(K)\right)\right)\right)
\end{aligned}
$$

and hence $\mathrm{Cl}\left(\operatorname{Int}^{\star}\left(F^{-}\left(\operatorname{Int}^{\star}(K)\right)\right)\right) \subseteq F^{-}(K)$.

$(2) \Rightarrow(3)$ : Let $K$ be any $\star$-closed set of $Y$. By (2) and Lemma $3(1)$,

$$
\begin{aligned}
& p \imath \mathrm{Cl}\left(F^{-}\left(\operatorname{Int}^{\star}(K)\right)\right) \\
& =F^{-}\left(\operatorname{Int}^{\star}(K)\right) \cup \mathrm{Cl}\left(\operatorname{Int}^{\star}\left(F^{-}\left(\operatorname{Int}^{\star}(K)\right)\right)\right) \\
& \subseteq F^{-}\left(\operatorname{Int}^{\star}(K)\right) \cup F^{-}(K)=F^{-}(K) .
\end{aligned}
$$

$(3) \Rightarrow(4)$ : The proof is obvious.

$(4) \Rightarrow(5)$ : Let $B$ be any subset of $Y$. Then by (4), we have

$$
\begin{aligned}
& X-p \imath \operatorname{Int}\left(F^{+}\left(\mathrm{Cl}^{\star}\left(\operatorname{Int}^{\star}(B)\right)\right)\right) \\
& =p \imath \mathrm{Cl}\left(X-F^{+}\left(\mathrm{Cl}^{\star}\left(\operatorname{Int}^{\star}(B)\right)\right)\right) \\
& =p \imath \mathrm{Cl}\left(F^{-}\left(Y-\mathrm{Cl}^{\star}\left(\operatorname{Int}^{\star}(B)\right)\right)\right) \\
& =p \imath \mathrm{Cl}\left(F^{-}\left(\operatorname{Int}^{\star}\left(\mathrm{Cl}^{\star}(Y-B)\right)\right)\right) \\
& \subseteq F^{-}\left(\mathrm{Cl}^{\star}(Y-B)\right) \\
& =X-F^{+}\left(\operatorname{Int}^{\star}(B)\right)
\end{aligned}
$$

and hence $F^{+}\left(\operatorname{Int}^{\star}(B)\right) \subseteq p \imath \operatorname{Int}\left(F^{+}\left(\mathrm{Cl}^{\star}\left(\operatorname{Int}^{\star}(B)\right)\right)\right)$.

$(5) \Rightarrow(1)$ : Let $V$ be any $\star$-open set of $Y$. Then by (5), we have $F^{+}(V) \subseteq p \imath \operatorname{Int}\left(F^{+}\left(\mathrm{Cl}^{\star}(V)\right)\right)$ and hence $F$ is upper almost weakly $\star$-continuous by Theorem 5 .

Theorem 8. For a multifunction $F:(X, \tau, \mathscr{I}) \rightarrow$ $(Y, \sigma, \mathscr{J})$, the following properties are equivalent:

(1) $F$ is lower almost weakly $\star$-continuous;

(2) $\operatorname{Cl}\left(\operatorname{Int} t^{\star}\left(F^{+}\left(\operatorname{Int}^{\star}(K)\right)\right)\right) \subseteq F^{+}(K)$ for every $\star-$ closed set $K$ of $Y$,

(3) $\operatorname{piCl}\left(F^{+}\left(\operatorname{Int}^{\star}(K)\right)\right) \subseteq F^{+}(K)$ for every $\star-$ closed set $K$ of $Y$,

(4) $\operatorname{plCl}\left(F^{+}\left(\operatorname{Int}^{\star}\left(C l^{\star}(B)\right)\right)\right) \subseteq F^{+}\left(C l^{\star}(B)\right)$ for every subset $B$ of $Y$;

(5) $F^{-}\left(\operatorname{In} t^{\star}(B)\right) \subseteq p l \operatorname{Int}\left(F^{-}\left(C l^{\star}\left(\operatorname{Int}^{\star}(B)\right)\right)\right)$ for every subset $B$ of $Y$.

Proof. The proof is similar to that of Theorem 7.
Definition 9. A function $f:(X, \tau, \mathscr{I}) \rightarrow(Y, \sigma, \mathscr{J})$ is said to be almost weakly $\star$-continuous if

$$
f^{-1}(V) \subseteq \operatorname{Int}\left(C l^{\star}\left(f^{-1}\left(C l^{\star}(V)\right)\right)\right)
$$

for each $\star$-open set $V$ of $Y$.

Corollary 10. For a function $f:(X, \tau, \mathscr{I}) \rightarrow$ $(Y, \sigma, \mathscr{J})$, the following properties are equivalent:

(1) $f$ is almost weakly $\star$-continuous;

(2) $C l\left(\operatorname{Int}^{\star}\left(f^{-1}(V)\right)\right) \subseteq f^{-1}\left(C l^{\star}(V)\right)$ for every $\star-$ open set $V$ of $Y$;

(3) $p \imath C l\left(f^{-1}(V)\right) \subseteq f^{-1}\left(C l^{\star}(V)\right)$ for every $\star$-open set $V$ of $Y$;

(4) $f^{-1}(V) \subseteq \operatorname{punt}\left(f^{-1}\left(C l^{\star}(V)\right)\right)$ for every $\star$-open set $V$ of $\bar{Y}$;

(5) for each $x \in X$ and each $\star$-open set $V$ of $Y$ containing $f(x)$, there exists a pre- $\mathscr{I}$-open set $U$ of $X$ containing $x$ such that $f(U) \subseteq C l^{\star}(V)$.

Definition 11. [4] $A$ point $x$ in an ideal topological space $(X, \tau, \mathscr{I})$ is called $a \star_{\theta}$-cluster point of $A$ if

$$
C l^{\star}(U) \cap A \neq \emptyset
$$

for every $\star$-open set $U$ of $X$ containing $x$. The set of all $\star_{\theta}$-cluster points of $A$ is called the $\star_{\theta}$-closure of $A$ and is denoted by $\star_{\theta} \mathrm{Cl}(A)$.

Definition 12. [4] A subset $A$ of an ideal topological space $(X, \tau, \mathscr{I})$ is called

(1) $\star_{\theta}$-closed if $\star_{\theta} \operatorname{Cl}(A)=A$.

(2) $\star_{\theta}$-open if its complement is $\star_{\theta}$-closed.

Lemma 13. [4] For a subset A of an ideal topological space $(X, \tau, \mathscr{I})$, the following properties hold:

(1) If $A$ is $\star$-open in $X$, then $C l^{\star}(A)=\star_{\theta} C l(A)$.

(2) $\star_{\theta} \mathrm{Cl}(A)$ is $\star$-closed in $X$.

Definition 14. [5] A subset $A$ of an ideal topological space $(X, \tau, \mathscr{I})$ is said to be:

(1) $R$ - $\mathscr{I}^{\star}$-open if $A=\operatorname{Int} t^{\star}\left(C l^{\star}(A)\right)$;

(2) $R-I^{\star}$-closed if its complement is $R-\mathscr{I}^{\star}$-open;

(3) $\mathscr{I}^{\star}$-preopen if $A \subseteq \operatorname{In} t^{\star}\left(C l^{\star}(A)\right)$;

(4) $\mathscr{I}^{\star}$-preclosed if its complement is $\mathscr{I}^{\star}$-preopen.

Theorem 15. For a multifunction $F:(X, \tau, \mathscr{I}) \rightarrow$ $(Y, \sigma, \mathscr{J})$, the following properties are equivalent:

(1) F is upper almost weakly $\star$-continuous; 
(2) $\operatorname{plCl}\left(F^{-}\left(\operatorname{Int} t^{\star}\left(\star_{\theta} C l(B)\right)\right)\right) \subseteq F^{-}\left(\star_{\theta} C l(B)\right)$ for every subset $B$ of $Y$;

(3) $\operatorname{plCl}\left(F^{-}\left(\operatorname{Int}^{\star}\left(C l^{\star}(V)\right)\right)\right) \subseteq F^{-}\left(C l^{\star}(V)\right)$ for every $\star$-open set $V$ of $Y$;

(4) $\operatorname{piCl}\left(F^{-}\left(\operatorname{Int}^{\star}\left(C l^{\star}(V)\right)\right)\right) \subseteq F^{-}\left(C l^{\star}(V)\right)$ for every $\mathscr{J}^{\star}$-preopen set $V$ of $Y$;

(5) $\operatorname{plCl}\left(F^{-}\left(\operatorname{Int} t^{\star}(K)\right)\right) \subseteq F^{-}(K)$ for every $R-\mathscr{J}^{\star}{ }_{-}$ closed set $K$ of $Y$.

Proof. (1) $\Rightarrow(2)$ : Let $B$ be any subset of $Y$. Suppose that $x \in X-F^{-}\left(\star_{\theta} \mathrm{Cl}(B)\right)$. Then, we have

$$
F(x) \subseteq Y-\star_{\theta} \operatorname{Cl}(B) .
$$

Since $Y-\star_{\theta} \mathrm{Cl}(B)$ is $\star$-open in $Y$ and by Theorem 5, there exists a pre- $\mathscr{I}$-open set $U$ of $X$ containing $x$ such that $F(U) \subseteq \mathrm{Cl}^{\star}\left(Y-\star_{\theta} \mathrm{Cl}(B)\right)$. Therefore,

$$
\begin{aligned}
U & \subseteq F^{+}\left(\operatorname{Cl}^{\star}\left(Y-\star_{\theta} \operatorname{Cl}(B)\right)\right) \\
& =F^{+}\left(Y-\operatorname{Int}^{\star}\left(\star_{\theta} \operatorname{Cl}(B)\right)\right) \\
& =X-F^{-}\left(\operatorname{Int}^{\star}\left(\star_{\theta} \operatorname{Cl}(B)\right)\right) .
\end{aligned}
$$

This implies that $U \cap F^{-}\left(\operatorname{Int}^{\star}\left(\star_{\theta} \operatorname{Cl}(B)\right)\right)=\emptyset$ and hence $x \in X-p \imath \mathrm{Cl}\left(F^{-}\left(\operatorname{Int}^{\star}\left(\star_{\theta} \operatorname{Cl}(B)\right)\right)\right)$. Consequently, we obtain

$$
p \imath \mathrm{Cl}\left(F^{-}\left(\operatorname{Int} \star^{\star}\left(\star_{\theta} \mathrm{Cl}(B)\right)\right)\right) \subseteq F^{-}\left(\star_{\theta} \mathrm{Cl}(B)\right) .
$$

$(2) \Rightarrow(3)$ : This follows from Lemma 13(1).

$(3) \Rightarrow(4)$ : Let $V$ be any $\mathscr{J}^{\star}$-preopen set of $Y$. Then $V \subseteq \operatorname{Int}^{\star}\left(\mathrm{Cl}^{\star}(V)\right)$ and by $(3)$,

$$
\begin{aligned}
& p \imath \mathrm{Cl}\left(F^{-}\left(\operatorname{Int}^{\star}\left(\mathrm{Cl}^{\star}(V)\right)\right)\right) \\
& =p \imath \mathrm{Cl}\left(F^{-}\left(\operatorname{Int}^{\star}\left(\mathrm{Cl}^{\star}\left(\operatorname{Int}^{\star}\left(\mathrm{Cl}^{\star}(V)\right)\right)\right)\right)\right) \\
& \subseteq F^{-}\left(\mathrm{Cl}^{\star}\left(\operatorname{Int}^{\star}\left(\mathrm{Cl}^{\star}(V)\right)\right)\right) \\
& =F^{-}\left(\mathrm{Cl}^{\star}(V)\right) .
\end{aligned}
$$

(4) $\Rightarrow(5)$ : Let $K$ be any $R-\mathscr{J}^{\star}$-closed set of $Y$. Since $\operatorname{Int}^{\star}(K)$ is $\mathscr{J}^{\star}$-preopen in $Y$ and by (4),

$$
\begin{aligned}
p \imath \mathrm{Cl}\left(F^{-}\left(\operatorname{Int}^{\star}(K)\right)\right) & =p \imath \mathrm{Cl}\left(F^{-}\left(\operatorname{Int}^{\star}\left(\mathrm{Cl}^{\star}\left(\operatorname{Int}^{\star}(K)\right)\right)\right)\right) \\
& \subseteq F^{-}\left(\mathrm{Cl}^{\star}\left(\operatorname{Int}^{\star}(K)\right)\right)=F^{-}(K) .
\end{aligned}
$$

$(5) \Rightarrow(1)$ : Let $V$ be any $\star$-open set of $Y$. Then $\mathrm{Cl}^{\star}(V)$ is $R-\mathscr{J}^{\star}$-closed in $Y$ and by (5), we have

$$
\begin{aligned}
p \imath \mathrm{Cl}\left(F^{-}(V)\right) & \subseteq p \imath \mathrm{Cl}\left(F^{-}\left(\operatorname{Int}^{\star}\left(\mathrm{Cl}^{\star}(V)\right)\right)\right) \\
& \subseteq F^{-}\left(\mathrm{Cl}^{\star}(V)\right) .
\end{aligned}
$$

It follows from Theorem 5 that $F$ is upper almost weakly $\star$-continuous.

Theorem 16. For a multifunction $F:(X, \tau, \mathscr{I}) \rightarrow$ $(Y, \sigma, \mathscr{J})$, the following properties are equivalent:
(1) F is lower almost weakly $\star$-continuous;

(2) $\operatorname{plCl}\left(F^{+}\left(\operatorname{Int} t^{\star}\left(\star_{\theta} C l(B)\right)\right)\right) \subseteq F^{+}\left(\star_{\theta} C l(B)\right)$ for every subset $B$ of $Y$,

(3) $\operatorname{plCl}\left(F^{+}\left(\operatorname{Int}^{\star}\left(C l^{\star}(V)\right)\right)\right) \subseteq F^{+}\left(C l^{\star}(V)\right)$ for every $\star$-open set $V$ of $Y$;

(4) $\mathrm{plCl}\left(\mathrm{F}^{+}\left(\operatorname{Int}^{\star}\left(C l^{\star}(V)\right)\right)\right) \subseteq \mathrm{F}^{+}\left(C l^{\star}(V)\right)$ for every $\mathscr{J}^{\star}$-preopen set $V$ of $Y$;

(5) $\operatorname{p\imath Cl}\left(F^{+}\left(\operatorname{Int}^{\star}(K)\right)\right) \subseteq F^{+}(K)$ for every $R-\mathscr{J}^{\star}$ closed set $K$ of $Y$.

Proof. The proof is similar to that of Theorem 15.

Corollary 17. For a function $f:(X, \tau, \mathscr{I}) \rightarrow$ $(Y, \sigma, \mathscr{J})$, the following properties are equivalent:

(1) $f$ is almost weakly $\star$-continuous;

(2) $\operatorname{p\imath Cl}\left(f^{-1}\left(\operatorname{Int} t^{\star}\left(\star_{\theta} C l(B)\right)\right)\right) \subseteq f^{-1}\left(\star_{\theta} C l(B)\right)$ for every subset $B$ of $Y$,

(3) $p \imath C l\left(f^{-1}\left(\operatorname{Int}^{\star}\left(C l^{\star}(V)\right)\right)\right) \subseteq f^{-1}\left(C l^{\star}(V)\right)$ for every $\star$-open set $V$ of $Y$;

(4) $\mathrm{plCl}\left(f^{-1}\left(\operatorname{Int^{\star }}\left(C l^{\star}(V)\right)\right)\right) \subseteq f^{-1}\left(C l^{\star}(V)\right)$ for every $\mathscr{J}^{\star}$-preopen set $V$ of $Y$;

(5) $\operatorname{p\imath Cl}\left(f^{-1}\left(\operatorname{Int} t^{\star}(K)\right)\right) \subseteq f^{-1}(K)$ for every $R$ $\mathscr{J}^{\star}$-closed set $K$ of $Y$.

\section{Conclusion}

The branch of mathematics called topology is concerned with all questions directly or indirectly related to continuity. Especially, the study of continuity has been found to be very useful in computer science and digital topology. Continuity of multifunctions in topological spaces has been researched by many mathematicians. This paper deals with the concepts of upper and lower almost weakly $\star$-continuous multifunctions in ideal topological spaces. Several characterizations of upper and lower almost weakly $\star$-continuous multifunctions are obtained. The ideas and results of this paper may motivate further research.

References: Abd El-Monsef, E. F. Lashien and A. A. Nasef, On $\mathscr{I}$-open sets and $\mathscr{I}$-continuous functions, Kyungpook Math. J. 32, 1992, pp. 2130.

[2] A. Açikgöz, T. Noiri and Ş. Yüksel, A decomposition of continuity in ideal topological spaces, Acta Math. Hungar. 105, 2004, pp. 285289. 
[3] C. Berge, Espaces topologiques fonctions multivoques, Dunod, Paris, 1959.

[4] C. Boonpok, Weak quasi continuity for multifunctions in ideal topological spaces, $A d v$. Math., Sci. J. 9(1), 2020, pp. 339-355.

[5] C. Boonpok, On continuous multifunctions in ideal topological spaces, Lobachevskii $J$. Math. 40, 2019, pp. 24-35.

[6] J. Dontchev, Idealization of Granter-Reilly decomposition theorems, arxiv:math. GN/9901017v1, 1999.

[7] E. Ekici, S. Jafari and V. Popa, On almost contra-continuous multifunctions, Lobachevskii J. Math. 30, 2009, pp. 124-131.

[8] E. Ekici, S. Jafari, M. Caldas and T. Noiri, Weakly $\lambda$-continuous functions, Novi Sad $J$. Math. 38, 2008, pp. 47-56.

[9] E. Hatir, A. Keskin and T. Noiri, A note on strong $\beta$ - $\mathscr{I}$-open sets and strongly $\beta-\mathscr{I}$ continuous functions, Acta Math. Hungar. 108, 2005, pp. 87-94.

[10] E. Hatir and T. Noiri, On semi- $\mathscr{I}$-open sets and semi- $\mathscr{I}$-continuous functions, Acta Math. Hungar. 107, 2005, pp. 345-353.

[11] E. Hatir and T. Noiri, On decompositions of continuity via idealization, Acta Math. Hungar. 96, 2002, pp. 341-349.

[12] T. Husain, Almost continuous mappings, Prace Mat. 10, 1966, pp. 1-7.

[13] D. S. Janković and T.-R. Hamlett, New topologies from old via ideals, Amer. Math. Monthly 97, 1990, pp. 295-310.

[14] D. S. Janković, $\theta$-regular spaces, Internat. $J$. Math. Math. Sci. 8, 1985, pp. 615-619.

[15] K. Kuratowski, Topology, Vol. I, AcademicPress, New York, 1966.

[16] N. Levine, A decomposition of continuity in topological spaces, Amer. Math. Monthly 68, 1961, pp. 44-46.

[17] T. Noiri and V. Popa, A unified theory of almost continuity for multifunctions, Sci. Stud. Res. Ser. Math. Inform. 20, 2010, pp. 185-124.
[18] T. Noiri and V. Popa, Almost weakly continuous multifunctions, Demonstratio Math. 26, 1993, pp. 363-380.

[19] V. Popa and T. Noiri, On weakly $(\tau, m)$ continuous functions, Rend. Circ. Mat. Palermo 51, 2002, pp. 295-316.

[20] V. Popa, Weakly continuous multifunctions, Boll. Un. Mat. Ital. (5), 15-A, 1978, pp. 379388.

[21] V. Popa, On certain properties of quasi continuous and almost continuous multifunctions (Romanian), Stud. Circ. Mat. 30, 1978, pp. 441446.

[22] R. E. Smithson, Almost and weak continuity for multifunctions, Bull. Calcutta Math. Soc. 70, 1978, pp. 383-390.

[23] V. Vaidyanathswamy, The localization theory in set topology, Proc. Indian Acad. Sci. 20, 1945, pp. 51-61.

[24] Ş. Yüksel, A. Açikgöz, and E. Gursel, On a new type of continuous functions in ideal topological spaces, J. Indian Acad. Math. 28, 2007, pp. 427438.

[25] Ş. Yüksel, T. H. Simsekler, Z. Guzel and T. Noiri, Strongly $\theta$-pre- $\mathscr{I}$-continuous functions, Sci. Stud. Res. Ser. Math. Inform. 20, 2010, pp. 111-126.

\section{Creative Commons Attribution License 4.0 (Attribution 4.0 International , CC BY 4.0)}

This article is published under the terms of the Creative Commons Attribution License 4.0

https://creativecommons.org/licenses/by/4.0/deed.en US 